\title{
Brain Abscess in a Patient With Decompensated Cirrhosis
}

\author{
Jun Kato ${ }^{\mathrm{a}}$, Masahiko Koda ${ }^{\mathrm{a}, \mathrm{b}}$, Yuki Fujise ${ }^{\mathrm{a}}$, Shiho Tokunaga ${ }^{\mathrm{a}}$, Tomomitsu Matono ${ }^{\mathrm{a}}$, \\ Takaaki Sugihara $^{a}$, Takakazu Nagahara ${ }^{a}$, Yoshikazu Murawaki ${ }^{a}$
}

\begin{abstract}
Brain abscess is rare but life-threatening, especially in a patient with decompensated cirrhosis. A 56-year-old woman with hepatitis B-related decompensated cirrhosis was admitted for disturbance of consciousness and high fever. She had right hemiparesis and mild neck stiffness, but no flapping tremor. Brain CT showed a low-density area in the left lenticular nucleus, indicating a brain abscess. She was given meropenem and ampicillin intravenously. Although the brain abscess improved, she died of progressive hepatic failure. In such patients, brain abscesses need to be distinguished from hepatic encephalopathy and treated quickly. Nevertheless, brain abscess in decompensated cirrhosis is extremely serious.
\end{abstract}

Keywords: Brain abscess; Liver cirrhosis; Sepsis; Disturbance of consciousness

\section{Introduction}

Brain abscess is a life-threatening disease with a poor prognosis that often develops as an opportunistic infection in patients with impaired host defense mechanism due to malignancy, diabetes mellitus, alcoholism, and liver cirrhosis [1, 2]. Brain abscess develops either by spread from a contiguous focus or by hematogenous spread from a distant focus, such as intra-abdominal infection, endocarditis, urinary tract infection, or pulmonary infection.

\section{Manuscript accepted for publication January 13, 2011}

${ }^{\mathrm{a}}$ Second Department of Internal Medicine, Tottori University, School of Medicine, Yonago 683-8504, Japan

${ }^{b}$ Corresponding author: Masahiko Koda, Second Department of Inter nal Medicine, Tottori University, School of Medicine, Nishi-cho 36-1, Yonago 683-8504, Japan. Email: masakoda@grape.med.tottori-u.ac.jp

doi:10.4021/jmc128w
Liver cirrhosis is an immune-compromised state because of reticuloendothelial dysfunction and porto-systemic shunting [3]. Bacterial infections in cirrhosis are common, particularly in decompensated patients, and they account for significant mortality [4]. A case of brain abscess in a decompensated cirrhotic patient is reported.

\section{Case Report}

A 56-year-old woman had hepatitis B virus-related cirrhosis (Child-Pugh class C) and was medicated with Lamivudine. She was admitted to the emergency department in our hospital for disturbance of consciousness and high fever in May 2009. She had a history of previous episodes of hepatic encephalopathy and a small hepatocellular carcinoma treated by ethanol injection 2 years ago. She had no history of head trauma, contiguous infection, or diabetes mellitus. On admission, her level of consciousness was Glasgow Coma Scale score 9 (E1V4M4). Right hemiparesis and mild neck stiffness were suspected, but there was no flapping tremor. Her blood pressure was $120 / 50 \mathrm{mmHg}$, pulse rate 127/ minute, and body temperature $39.4{ }^{\circ} \mathrm{C}$. The laboratory test results were: total white blood cell count $15,400 / \mathrm{mm}^{3}$ with 93\% neutrophils, C-reactive protein $1.26 \mathrm{mg} / \mathrm{dl}$, hemoglobin $8.4 \mathrm{~g} / \mathrm{dl}$, platelet count $60,000 / \mathrm{mm}^{3}$, prothrombin time $42.0 \%$, serum glucose $64 \mathrm{mg} / \mathrm{dl}$, albumin $2.0 \mathrm{~g} / \mathrm{dl}$, total bilirubin $5.9 \mathrm{mg} / \mathrm{dl}$, AST $47 \mathrm{IU} / \mathrm{L}$, ALT $23 \mathrm{IU} / \mathrm{L}, \mathrm{NH} 392 \mu \mathrm{g} / \mathrm{dl}$, alpha-fetoprotein $1.4 \mathrm{ng} / \mathrm{ml}$ and des- $\gamma$-carboxy prothrombin $17 \mathrm{mAU} / \mathrm{ml}$, hepatitis B surface-antigen positive, e-antigen negative and hepatitis B virus-DNA negative. Blood cultures were negative for bacteria.

Plain brain computed tomography (CT) revealed a low-density lesion in the left lenticular nucleus and caudate nucleus, compressing the left lateral ventricle (Fig. 1a). We suspected a brain abscess and administered antibiotic (meropenem $0.5 \mathrm{~g}$ every 8 hours, intravenously) empirically. Her clinical course is shown in Figure 2. Her consciousness improved promptly, but 3 days later, it again deteriorated, and signs of meningeal irritation appeared. Brain contrastenhanced CT showed an enlarged, low-density lesion with slight ring enhancement (Fig. 1b) [5]. To make a definite di- 


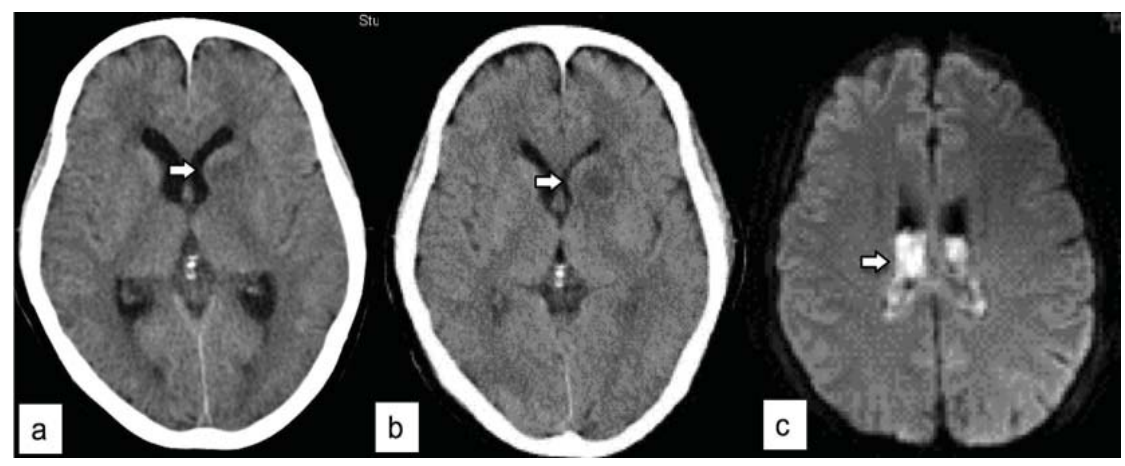

Figure 1. (a) Plain brain computed tomography reveals a low-density lesion (arrow) in the left lenticular nucleus and caudate nucleus, and the left lateral ventricle is compressed. (b) Brain contrast-enhanced CT shows enlarged low-density lesion (arrow) with slight ring enhancement. (c) Brain contrast-enhanced magnetic resonance imaging shows high-intensity areas (arrow) on diffusion-weighted images in bilateral lateral ventricles, indicating rupture of the abscess into the ventricles.

agnosis, lumbar puncture was performed after platelet transfusion. Cerebrospinal fluid (CSF) analysis showed pleocyto-

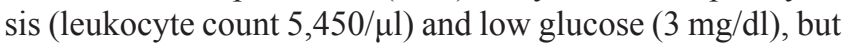
the CSF culture was negative for bacteria. A brain abscess with bacterial meningitis was diagnosed, and parenteral antibiotic therapy was intensified (meropenem 2 g every 8 hours, ampicillin 2 g every 4 hours). On the 6 th day, brain contrastenhanced magnetic resonance imaging (MRI) showed slight high-intensity areas on Fluid Attenuated Inversion Recovery and T1-weighted images, and high-intensity areas in bilateral lateral ventricles on diffusion-weighted images (Fig. 1c). These findings indicated bloody fluid and a brain abscess that ruptured into the ventricle. Thereafter, though her brain abscess improved, her hepatic failure progressed and the patient died on the 134th day.

\section{Discussion}

Cirrhotic patients are susceptible to several infectious diseases, such as septicemia, meningitis, pneumonia, urinary tract infection, and spontaneous bacterial peritonitis (SBP) [4]. Six (11\%) of 53 cases of brain abscess had liver cirrhosis [1]. Patients with liver cirrhosis presented with nonspecific symptoms, and all of them died soon. This high mortality in cirrhotic patients is associated with several factors. First, it is difficult to differentiate brain abscess from hepatic encephalopathy. Only $34 \%$ of patients have the complete triad, which is headache, fever, and focal neurologic deficits [1]. In the advanced stage of liver cirrhosis, hepatic encephalopathy is common and usually presents with disorientation, confusion, drowsiness, and coma; our patient had a past history of hepatic encephalopathy. Furthermore, cirrhotic patients can often have episodes of fever, caused by endotoxemia or SBP. Fortunately, in the present case, a neurologist suspected cranial infection because of mild neck stiffness and hemiparesis. However, neck stiffness occurs in only $8 \%$ of patients with brain abscess [1]. It is not always easy in clinical settings to detect mild neurological findings in patients with impaired consciousness. We should bear in mind the possibility of cranial infection when encountering cirrhotic patients with disturbance of consciousness and fever.

Second, bacterial infections worsen hepatic failure, and the prognosis is affected adversely even after resolution of infection $[6,7]$. With infection, a systemic inflammatory response syndrome may occur, resulting in sepsis, renal failure, encephalopathy, and death. Increased endotoxins, bile acids, nitric oxide, and cytokines, such as tumor necrosis factor- $\alpha$ and interleukin-6, lead to systemic, renal, and hepatic hemodynamic derangements. In the present case, hepatic failure progressed gradually after resolution of the brain abscess, resulting in death. It may be important to start treatment as quickly as possible to prevent systemic inflammatory responses. Third, most cirrhotic patients are not surgical candidates because of their bleeding tendency and low hepatic reserve. Only $33 \%$ of cirrhotic patients received surgical treatment, compared with $66 \%$ of non-cirrhotic patients [1].

Liver dysfunction leads to several abnormalities of defense mechanisms because of depressed humoral and cellmediated immunity. Bacterial translocation from the intestine induces bacteremia, and impaired hepatic bacterial clearance results in failure to control bacteremia [3]. In fact, some cases of brain abscess have been reported after endoscopic procedures, which induce bacteremia [8-10]. Severe bacteremia may cause infections of several organs, including brain abscess [4]. Furthermore, Klebsiella pneumoniae, which can colonize the gastrointestinal tract, is a common pathogen of brain abscess in cirrhotic patients [1, 11, 12]. In the present case, the pathogen causing the brain abscess could not be identified by blood and CSF cultures due to prior antibiotic therapy. Pathogenic bacteria are not identified in $30 \%$ of brain abscesses [1]. 


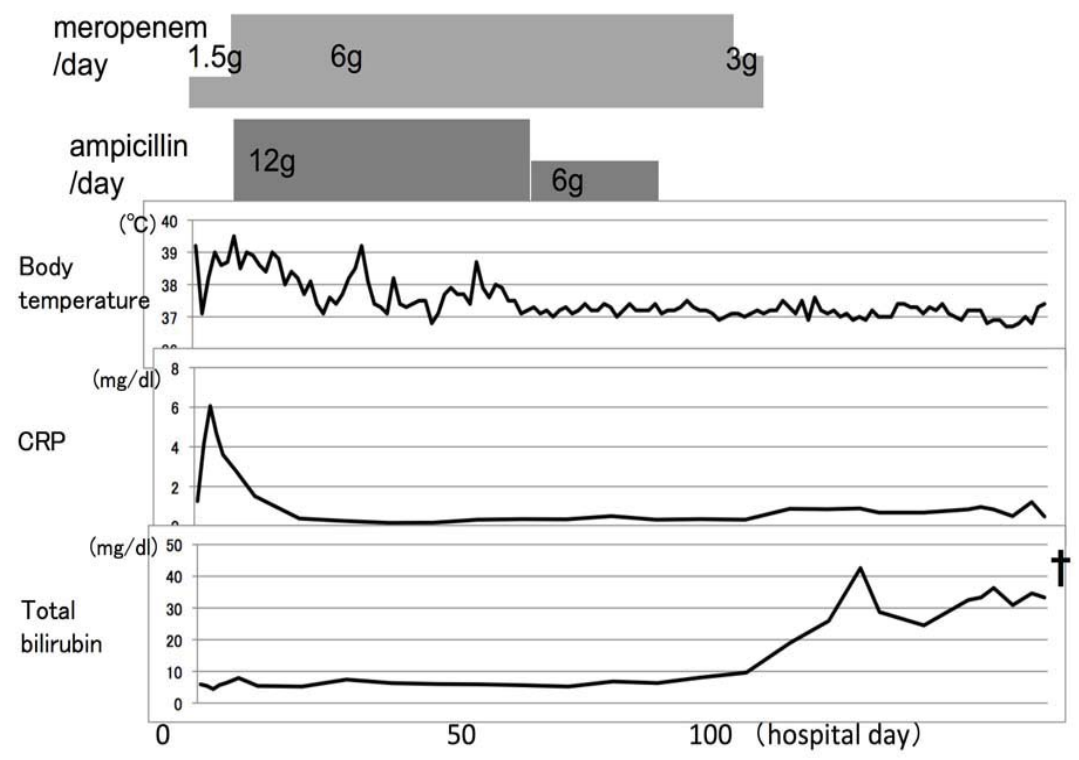

Figure 2. Clinical course.

In conclusion, cirrhotic patients with central nerve system (CNS) infection could be easily misdiagnosed or diagnosed late because hepatic encephalopathy is a common complication of liver cirrhosis. When dealing with cirrhotic patients with impaired consciousness and fever, brain CT or MRI must be considered to exclude the presence of CNS lesions.

\section{References}

1. Kao PT, Tseng HK, Liu CP, Su SC, Lee CM. Brain abscess: clinical analysis of 53 cases. J Microbiol Immunol Infect 2003;36(2):129-136.

2. Lu CH, Chang WN, Lui CC. Strategies for the management of bacterial brain abscess. J Clin Neurosci 2006;13(10):979-985.

3. Ashare A, Monick MM, Powers LS, Yarovinsky T, Hunninghake GW. Severe bacteremia results in a loss of hepatic bacterial clearance. Am J Respir Crit Care Med 2006;173(6):644-652.

4. Merli M, Lucidi C, Giannelli V, Giusto M, Riggio O, Falcone M, Ridola L, et al. Cirrhotic patients are at risk for health care-associated bacterial infections. Clin Gastroenterol Hepatol 2010;8(11):979-985.

5. Anslow P. Cranial bacterial infection. Eur Radiol 2004;14 Suppl 3:E145-154.

6. Arvaniti V, D’Amico G, Fede G, Manousou P, Tso- chatzis E, Pleguezuelo M, Burroughs AK. Infections in patients with cirrhosis increase mortality four-fold and should be used in determining prognosis. Gastroenterology 2010;139(4):1246-1256, 1256 e1241-1245.

7. Kim JH, Lee JS, Lee SH, Bae WK, Kim NH, Kim KA, Moon YS. Renal Dysfunction Induced by Bacterial Infection other than Spontaneous Bacterial Peritonitis in Patients with Cirrhosis: Incidence and Risk Factor. Gut Liver 2009;3(4):292-297.

8. Shih HI, Lee HC, Chuang CH, Ko WC. Fatal Klebsiella pneumoniae meningitis and emphysematous brain abscess after endoscopic variceal ligation in a patient with liver cirrhosis and diabetes mellitus. J Formos Med Assoc 2006;105(10):857-860.

9. Laviv Y, Ben-Daviv U, Vated M, Rappaport ZH. Brain abscess following endoscopic ligation of esophageal varicose veins. Acta Neurochir (Wien) 2010;152(4):733734.

10. Banerjee S, Shen B, Baron TH, Nelson DB, Anderson MA, Cash BD, Dominitz JA, et al. Antibiotic prophylaxis for GI endoscopy. Gastrointest Endosc 2008;67(6):791798.

11. Chung CL, Lieu AS, Chen IY, Kwan AL, Howng SL. Brain abscess in adult cirrhotic patients: two case reports. Kaohsiung J Med Sci 2007;23(1):34-39.

12. Cho KT, Park BJ. Gas-forming brain abscess caused by Klebsiella pneumoniae. J Korean Neurosurg Soc 2008;44(6):382-384. 